\title{
News And VIeWs
}

\section{EphB2: a signature of colorectal cancer stem cells to predict relapse}

\author{
Xiaoxue Zhang
}

Colorectal cancer is one of the most commonly diagnosed cancers and is the second leading cause of death from cancer. Currently, the conventional treatment in clinics is surgery combined with chemotherapy or radiation therapy. Although new drugs have been developed, relapse and metastasis occur in nearly half of colorectal cancer patients. As most other types of cancer, the recurrence rate of colorectal cancer is negatively correlated with the disease stage at diagnosis. Therefore, early diagnosis becomes an important approach to improve the treatment outcome. Consistent with this idea, prediction of the disease progression and recurrence has significant implications for the choices and outcome of treatment. With the rapid development of molecular biology, biochemistry and oncology in the past decade, scientists have taken great efforts identifying gene and/or biochemical markers that are associated with tumorigenesis, and several screening approaches have been developed and used in clinic. These applications have greatly benefited the early detection and remarkably reduced cancer deaths. Recently, Batlle group from Spain identified EphB2 as an intestinal stem cell (ISC) marker gene that can be used to identify colorectal cancer stem cells and predict colorectal cancer relapse (Merlos-Suárez et al., 2011).

ISCs reside at the base of mucosal invaginations (crypt). Their progeny transient amplifying cells move upward as they proliferate through mitosis until get close to intestinal lumen, where cells undergo cell cycle arrest and terminal differentiation. ISCs are essential for the tissue regeneration in intestine. However, they also exhibit relatively high efficiency to develop colorectal tumors upon mutations of genes in Wnt signaling pathway, which are suggested to activate intestinal tumorigenesis. EphB2 is a receptor tyrosine kinase that is regulated by Wnt pathway. Targeted deletion of EphB2, EphB3 or their ligand ephrin-B1 in mouse caused intestinal cell compartmentalization defects (Batlle et al., 2002; Cortina et al., 2007), indicating the essential role of EphB signaling in cell positioning in crypts.

EphB2 is expressed in a decreasing trend from the crypt base toward the differentiated cell compartment in intestine lumen, corresponding to intestinal stem cell marker, Lgr5
(Batlle et al., 2002). According to this distinct expression pattern, Batlle group purified ISCs (EphB2 $\left.{ }^{\text {hi }}\right)$, transient amplifying cells (EphB2 ${ }^{\text {med }}$ ) and separated cells $\left(E p h B 2^{l o}\right)$ from mouse small intestine. Gene expression profiling revealed high level of differentiation markers in EphB2 ${ }^{\text {low }}$ cells, but EphB2 ${ }^{\text {hi }}$ cells and EphB2 ${ }^{\text {med }}$ cells expressed high level of proliferating markers as well as mediators of DNA replication, mitosis and DNA-damage repair; EphB2 ${ }^{\text {hi }}$ cells also had stronger expression of stem cell markers than EphB2 ${ }^{\text {med }}$ cells. These expression profiles from mouse intestinal cells were validated in primary human colorectal cancer samples through investigation of publicly available transcriptomic data of normal human colon mucosa (Kosinski et al., 2007). In vitro culture study proved that EphB2 ${ }^{\text {hi }}$ cells were able to form complex multicellular 3D structures that contained differentiated intestinal cell types, but EphB2 ${ }^{\text {med }}$ cells displayed lower efficiency in forming such organoids, and EphB2 ${ }^{\mathrm{lo}}$ cells were not able to develop organoids at all. Both gene expression analysis and functional test supported the correlation of EphB2 ${ }^{\text {hi }}$ cells and ISCs (Merlos-Suárez et al., 2011).

Global transcriptional profile of human colorectal cancer samples showed that colorectal cancer cells exhibited high expression of crypt proliferation genes and down-regulation of intestinal differentiation marker genes in comparison to the normal tissue. $38 \%$ of EphB2 ${ }^{\text {hi }}$ ISC gene program was also overexpressed in colorectal cancer cells. Importantly, the expression of EphB2-ISC profile was stronger in the metastatic colorectal cancer than the non-metastatic counterpart, and the expression was also stronger in the malignant poorlydifferentiated tumors than in benign well-differentiated tumors. In addition, the investigation of ISC gene expression in colorectal cancer patients indicated that the EphB2-derived ISC signature is highly correlated with the risk of colorectal cancer recurrence, especially the late relapse that occurred after 2 years of curative therapy. These findings have suggested that EphB2 ${ }^{\text {hi }}$ ISC gene signature is positively correlated with colorectal cancer aggressiveness and can be a predictor for colorectal cancer relapse (Merlos-Suárez et al., 2011).

Finally, the study provided evidence that EhpB2 is an 
effective surface marker for ISC-like tumor cells. Subcutaneous injection of EphB2-purified tumor cells into NOD/SCID mice revealed significantly higher tumorigenic capacity for $E_{\text {phB2 }}{ }^{\text {hi }}$ tumor cells than for EphB2 ${ }^{\text {lo }}$ tumor cells, and EphB2 ${ }^{\text {med }}$ tumor cells had intermediate capacity; while the three types of tumor cells had similar viability before injection. Further analysis showed that the xenografts generated by EphB2 ${ }^{\text {hi }}$ tumor cells exhibited similar histological patterns as primary tumors. The EphB2 ${ }^{\text {hi }}$ cells from secondary xenograft had similar expression profiles of differentiation, proliferation and ISC marker genes to the corresponding EphB2 ${ }^{\text {hi }}$, EphB2 $^{\text {lo }}$ and EphB2 ${ }^{\text {med }}$ cells in primary xenograft. These results showed that the EphB2 ${ }^{\text {hi }}$ ISC-like tumor cells display great tumorigenic potential with self-renewal and differentiation capacity (Merlos-Suárez et al., 2011).

This study by Merlos-Suárez et al. (2011) not only characterized EphB2 as an ISC signature that can be used to identify ISC-like tumor cells and to predict colorectal cancer relapse, but also established a link between tumor-initiating cells and cancer recurrence. The high risk of tumor regeneration in the presence of EphB2 ${ }^{\text {hi }}$ ISC-like tumor cells suggests EphB2 and ISC function as potential therapeutic targets to prevent and control colorectal cancer recurrence. In addition, these findings may provide insights into the understanding and treatment of other cancers.

\section{REFERENCES}

Batlle, E., Henderson, J.T., Beghtel, H., van den Born, M.M., Sancho, E., Huls, G., Meeldijk, J., Robertson, J., van de Wetering, M., Pawson, T., et al. (2002). Beta-catenin and TCF mediate cell positioning in the intestinal epithelium by controlling the expression of EphB/ephrinB. Cell 111, 251-263.

Cortina, C., Palomo-Ponce, S., Iglesias, M., Fernández-Masip, J.L., Vivancos, A., Whissell, G., Humà, M., Peiró, N., Gallego, L., Jonkheer, S., et al. (2007). EphB-ephrin-B interactions suppress colorectal cancer progression by compartmentalizing tumor cells. Nat Genet 39, 1376-1383.

Kosinski, C., Li, V.S., Chan, A.S., Zhang, J., Ho, C., Tsui, W.Y., Chan, T.L., Mifflin, R.C., Powell, D.W., Yuen, S.T., et al. (2007). Gene expression patterns of human colon tops and basal crypts and BMP antagonists as intestinal stem cell niche factors. Proc Natl Acad Sci U S A 104, 15418-15423.

Merlos-Suárez, A., Barriga, F.M., Jung, P., Iglesias, M., Céspedes, M. V., Rossell, D., Sevillano, M., Hernando-Momblona, X., da SilvaDiz, V., Muñoz, P., et al. (2011). The intestinal stem cell signature identifies colorectal cancer stem cells and predicts disease relapse. Cell Stem Cell 8, 511-524. 